\title{
Influence de la taille des vitroplants et du type d'explant sur la réponse à la callogenèse chez le cotonnier (Gossypium hirsutum L.) cultivé en Côte d'Ivoire
}

\author{
Hilaire Tanoh KOUAKOU ${ }^{1 *}$, Laurent Kouakou KOUAKOU ${ }^{1}$, Modeste Kan KOUASSI ${ }^{2}$, \\ Edmond Kouablan KOFFI ${ }^{2}$ et Mongomaké KONE ${ }^{1}$
}

${ }^{I}$ Laboratoire de Biologie et Amélioration des Productions Végétales, UFR Sciences de la Nature, Université d'Abobo-Adjamé, 02 BP 801 Abidjan 02, Côte d'Ivoire.

${ }^{2}$ Laboratoire Central de Biotechnologies, Centre National de Recherche Agronomique, 01 P. O. Box 1740 Abidjan 01, Côte d'Ivoire.

*Auteur correspondant ; E-mail: tanohilaire@yahoo.fr; Tel :(225) 20-37-81-21,

Fax : (225) 20-37-81-18

\section{RESUME}

L'aptitude à la callogenèse chez le cotonnier a été estimée par le taux d'induction et le poids sec des cals en fonction de la source de l'explant et de la taille des plantules. Le taux d'induction des cals augmente avec la taille des plantules alors que le poids sec des cals ; résultat de la croissance des cals, évolue en sens contraire. L'hypocotyle permet d'induire une bonne callogenèse suivi de la racine et du cotylédon quelle que soit la taille des plantules. Les plantules de petite taille ont une bonne aptitude à la callogenèse pour les explants hypocotyles et racines alors que pour les explants cotylédons, ce sont les plantules de grande taille qui favorisent l'induction des cals.

() 2014 International Formulae Group. All rights reserved.

Mots clés: Cal, explant, cotonnier, Gossypium hirsutum, vitroplants.

\section{INTRODUCTION}

Gossypium hirsutum L. est la principale espèce de cotonnier actuellement cultivée dans le monde (Kouadio et al., 2004). Il est cultivé pour ses fibres (coton) qui constituent la principale matière première en industrie textile et ses graines utilisées en alimentation humaine et animale (Sément, 1986). Les Etats-Unis, la Chine, l'Inde, l'Ouzbékistan et le Pakistan sont les plus grands producteurs, avec plus de $60 \%$ de la production mondiale. Les pays africains réunis participent pour $6 \%$ à cette production et la Côte d'Ivoire, avec près de 400000 tonnes de coton-graines, est le quatrième producteur africain au sud du Sahara après le Mali, le Bénin et le Burkina Faso (Anonyme, 2000). Le coton est donc une importante source de revenu pour la Côte d'Ivoire où il représente le troisième produit d'exportation après le cacao et le café (Kouakou, 2009).

Le coton représente une importante source de revenu pour les pays producteurs et constitue un peu plus de $50 \%$ du marché des fibres textiles (Anonyme, 2006). Cette place prédominante du coton est menacée par le 
développement des fibres synthétiques (30\%) et des fibres animales (19\%). En plus, la production, la qualité des fibres et des graines du cotonnier sont affectées par les attaques des parasites et des maladies diverses (Vaissayre et Lançon, 1991). Face à ces problèmes, l'hybridation interspécifique qui permet l'introduction de nouveaux caractères agronomiques s'avère être un outil intéressant pour l'amélioration variétale (Démol et al., 1992). Cependant, l'amélioration variétale par croisements interspécifiques a connu peu de succès chez le cotonnier à cause des barrières d'incompatibilités dues au niveau de ploïdie différents et et du temps relativement long pour obtenir une variété améliorée (Munro, 1987; Mergeai, 1992). Les difficultés rencontrées en croisement interspécifique et l'intérêt du coton sur le plan économique ont amené les chercheurs à initier la culture in vitro des tissus chez cette plante. La culture in vitro permet de contourner ces difficultés, dans la mesure où les cals obtenus à partir d'explants constituent un matériel adéquat pour la transformation génétique (Finer et Mc Mullen, 1990) et aussi un matériel idéal de départ pour d'autres vitrocultures comme la culture de protoplastes, les suspensions cellulaires et l'embryogenèse somatique (Olesen et al., 1995 ; Gonzalez-Benito et al., 1997 ; Blanc et al., 2002 ; Kouakou et al., 2004).

Chez le cotonnier, plusieurs types d'explants ont été utilisés pour l'initiation des cals. Généralement, ce sont les hypocotyles (Zouzou et al., 2000 ; Kouadio et al., 2004 ; Kouakou et al., 2006) qui ont été utilisés pour l'obtention des cals chez le cotonnier. Toutefois, quelques études ont rapporté l'utilisation chez le cotonnier des explants cotylédons et racines (Zouzou et al., 1997 ; Zhang et al., 2001). Les résultats obtenus à partir de ces expériences montrent que la callogenèse diffère d'un explant à l'autre. De plus, les explants sont utilisés sans tenir compte de la taille des plantules alors qu'une hétérogénéité de taille des plantules est observée après la germination in vitro des graines de cotonnier (Koné, 1996 ; Kouakou et al., 2004).

La taille des plantules et la source de l'explant influencent-elles la réponse à la callogenèse; ou bien existe-t-il une relation entre la taille des plantules, la source de l'explant et la callogenèse ? Ces interrogations et l'importance du cal, matériel de choix pour les transformations biotechnologiques, nous ont donc amené à réaliser cette étude afin d'optimiser la callogenèse chez le cotonnier.

\section{MATERIEL ET METHODES Matériel végétal}

Le matériel végétal est constitué d'hypocotyles, de cotylédons et de racines obtenus après la germination in vitro des graines de cotonnier (Gossypium hirsutum L.), cultivar R405-2000. Les graines, originaires de la Côte d'Ivoire ont été fournies par le Centre National de Recherche Agronomique (CNRA).

\section{Méthodes \\ Stérilisation et germination in vitro des graines}

Les graines sont stérilisées sous une hotte à flux laminaire. Elles sont trempées dans l'éthanol $70 \%(\mathrm{v} / \mathrm{v})$ pendant $1 \mathrm{~min}$, puis dans l'hypochlorite de sodium $2,5 \%$ de chlore actif pendant $20 \mathrm{~min}$, avec au besoin une goutte de Tween 20 pour $100 \mathrm{ml}$ de solution. Les graines sont ensuite rincées trois fois à l'eau distillée stérile et imbibées dans de l'eau distillée, stérilisée pendant $48 \mathrm{~h}$ à l'obscurité pour faciliter la germination. Elles sont débarrassées de leurs téguments et mises à germer dans des tubes à essais en verre Pyrex de dimension 150x22 ( $\mathrm{L}$ x $\varnothing$ en $\mathrm{mm}$ ) et de capacité $50 \mathrm{ml}$. La germination se fait sur le milieu de base MS (Murashige et Skoog, 1962) supplémenté de vitamine B5 (Gamborg et al., 1968) dont les macro et micro éléments ont été dilués de moitié. Ce milieu renferme également $30 \mathrm{~g} / \mathrm{l}$ de saccharose et $0,75 \mathrm{~g} / \mathrm{l}$ de $\mathrm{MgCl}_{2}$ et solidifié avec 2,5 g/l de gelrite. Les 
tubes à essais sont enfin hermétiquement fermés avec des bouchons plastiques de dimension $25 \times 38$ ( $\varnothing \times \mathrm{L}$ en $\mathrm{mm}$ ). Le $\mathrm{pH}$ du milieu de germination est ajusté à 5,8 avec du $\mathrm{NaOH}$ et/ou du $\mathrm{HCl}$.

\section{Induction des cals}

Sept jours après la germination, les plantules ont été classées selon leur taille (du collet à l'insertion des cotylédons). Les segments d'hypocotyle et de racine longs d'environ $5 \mathrm{~mm}$ et des carrés de cotylédon d'environ $5 \mathrm{~mm}$ de côté, ont été prélevés sur chaque lot de plantules et utilisés comme explants. Cinq explants ont été placés dans des boîtes de Pétri sur le milieu de callogenèse qui est un milieu de base MS contenant de la vitamine $\mathrm{B} 5$ auquel est ajouté $30 \mathrm{~g} / \mathrm{l}$ de glucose, 0,75 g/l de $\mathrm{MgCl}_{2}, 0,1 \mathrm{mg} / \mathrm{l}$ d'acide 2,4 dichlorophénoxyacétique (2,4-D) et 0,5 $\mathrm{mg} / \mathrm{l}$ de kinétine. Six boîtes de Pétri ont été utilisées par traitement.

Les milieux ont été stérilisés à l'autoclave pendant 30 min sous 1 bar à 121 ${ }^{\circ} \mathrm{C}$. Toutes les cultures ont été incubées dans une chambre de culture à une température de $28 \pm 2{ }^{\circ} \mathrm{C}$, sous une intensité lumineuse de 2000 lux fournie par des lampes blanches fluorescentes et une photopériode de $16 \mathrm{~h}$.

Après un mois de culture, la callogenèse a été estimée par le pourcentage de cals, représentant le degré d'initiation des cals et le poids sec (mg) des cals. Chaque valeur représente la moyenne de trois expériences distinctes. Chaque expérience ou traitement (taille-explant) comporte trente explants par répétition, soit un effectif total de quatre-vingt-dix explants.

\section{Analyses statistiques}

L'efficacité des explants à l'induction des cals a été évaluée par des analyses statistiques. Les données ont été analysées en utilisant le logiciel STATISTICA 6.0. En effet, avant toutes analyses, le test Kolmogorov-Smirnov pour vérifier la distribution normale des moyennes est effectué. Lorsque cette distribution n'est pas significative $(\mathrm{P}>0,001)$, aucune transformation n'est effectuée. Le test de Kruskal-Wallis est effectué dans le but de déterminer les différences significatives $(\mathrm{P}<$ $0,05)$ entre les traitements.

\section{RESULTATS}

\section{Germination in vitro des graines}

Après sept jours d'incubation, les plantules ont été obtenues après la germination. Suivant leur taille (Figure 1), trois lots de plantules ont été établis:

- Les plantules dont la taille est supérieure à $6 \mathrm{~cm} \mathrm{ou} \mathrm{P}_{6}$; la racine principale a une longueur moyenne de $9 \mathrm{~cm}$; l'hypocotyle a une longueur moyenne de 7,66 $\mathrm{cm}$ et un diamètre moyen de $0,38 \mathrm{~cm}$;

- Les plantules dont la taille est comprise entre 4 et $6 \mathrm{~cm}$ ou $\mathrm{P}_{46}$; la racine principale a une longueur moyenne de $5 \mathrm{~cm}$; l'hypocotyle a une longueur moyenne de $5,15 \mathrm{~cm}$ et un diamètre moyen de $0,42 \mathrm{~cm}$;

- Les plantules dont la taille est inférieure à $4 \mathrm{~cm}$ ou $\mathrm{P}_{4}$; la racine principale a une longueur moyenne de $2 \mathrm{~cm}$; l'hypocotyle a une longueur moyenne de 2,96 cm et est en général plus gros avec un diamètre moyen de $0,54 \mathrm{~cm}$.

Il faut noter qu'aucune différence morphologique significative n'a été observée au niveau des feuilles cotylédonaires des trois lots de plantules obtenues.

\section{Callogenèse}

Après un mois de culture, les explants ont initié des cals (Figure 2). Le pourcentage d'explants induisant des cals est significativement plus important avec les explants hypocotyles, quelle que soit la taille, que les autres explants. Les explants hypocotyles sont suivis des racines. Les plantules $\quad \mathrm{P}_{6}$ fournissent les meilleurs pourcentages d'induction de cals quel que soit le type d'explant utilisé, suivies des plantules $\mathrm{P}_{46}$ puis des plantules $\mathrm{P}_{4}$ (Tableau 1). 
Au niveau de la biomasse, les résultats indiquent que le poids sec des cals est significativement plus important avec les hypocotyles qu'avec les deux autres explants, quelle que soit leur taille. Cependant, les racines présentent un poids sec de cals plus élevé que les cotylédons. Au niveau des lots de plantules, le poids sec des cals est inversement proportionnel à la taille des plantules avec les explants hypocotyles et racines (Tableau 2).

$\mathrm{Au}$ total, nous constatons que les explants hypocotyles répondent mieux à la callogenèse que les racines suivies des cotylédons.

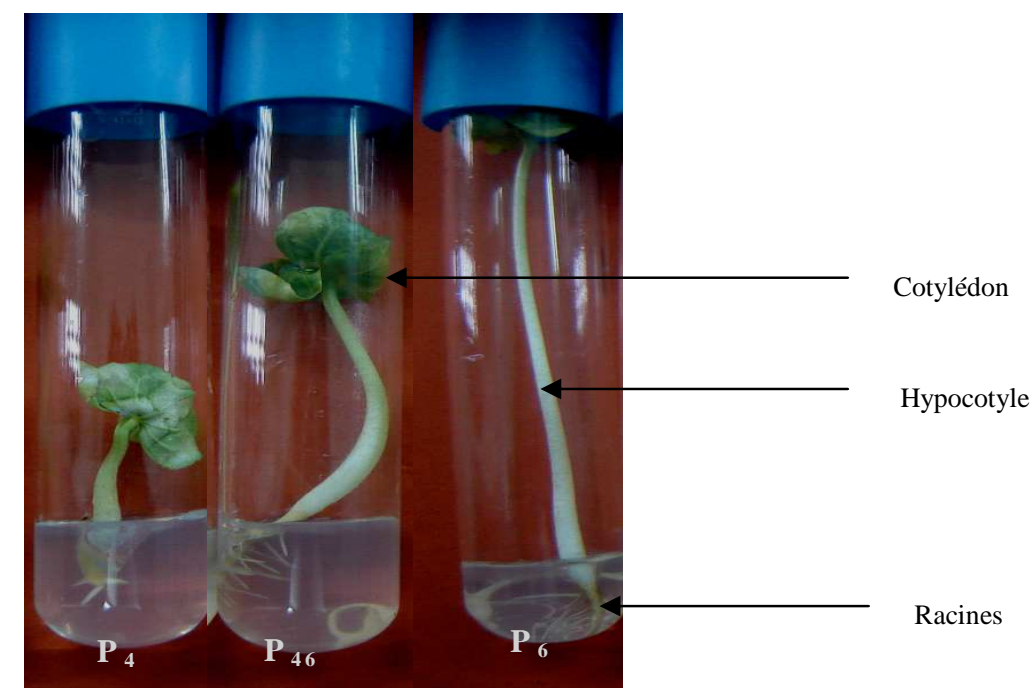

Figure 1 : Vitroplants de cotonnier âgés de sept jours. $\mathrm{P}_{4}, \mathrm{P}_{46}$ et $\mathrm{P}_{6}$ représentent respectivement les vitroplants dont la taille est: inférieure à $4 \mathrm{~cm}$, comprise entre 4 et $6 \mathrm{~cm}$ et supérieure à $6 \mathrm{~cm}$.

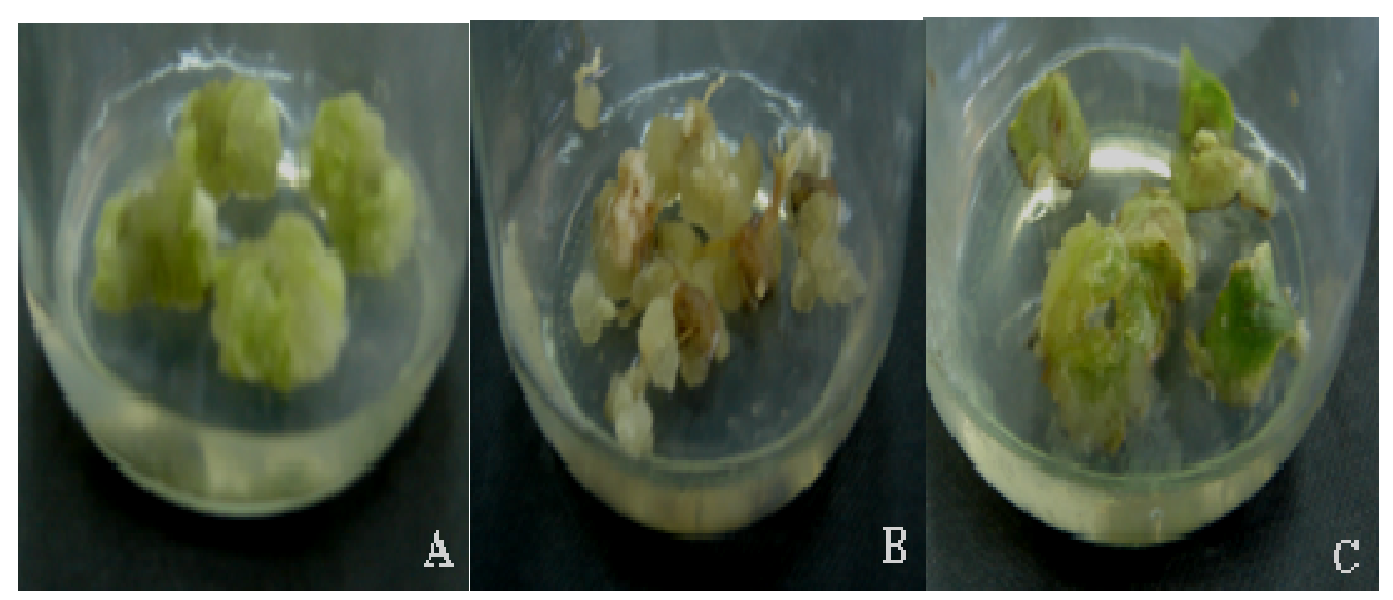

Figure 2 : Cals produits par les différents explants après un mois de culture chez le cotonnier. A, B et $\mathrm{C}$ représentent respectivement les cals provenant des explants d'hypocotyle, de racine et de cotylédon. 
Tableau 1 : Effet de la taille et du type d'explants sur le pourcentage d'induction des calls chez le cotonnier.

\begin{tabular}{lccc}
\hline \multirow{2}{*}{$\begin{array}{l}\text { Taille des } \\
\text { explants }\end{array}$} & \multicolumn{3}{c}{ Pourcentage d'induction de cals } \\
\cline { 2 - 4 } & \multicolumn{3}{c}{ Type d'explant } \\
\cline { 2 - 4 } & Hypocotyle & Racine & Cotylédon \\
\hline $\mathbf{P}_{\mathbf{4}}$ & $70,44 \pm 1,40^{\mathbf{c}}$ & $65,64 \pm 1,71^{\text {cd }}$ & $46,50 \pm 1,10^{\mathbf{e}}$ \\
$\mathbf{P 4 6}_{\text {P6 }}$ & $85,66 \pm 3,10^{\text {ab }}$ & $78,83 \pm 1,47^{\mathbf{b c}}$ & $55,88 \pm 1,38^{\mathbf{d}}$ \\
\hline Statistiques & $99,91 \pm 1,95^{\mathbf{a}}$ & $90,12 \pm 1,90^{\text {ab }}$ & $80,83 \pm 1,87^{\mathbf{b}}$ \\
\hline & $\mathrm{F}=38,46$ & $\mathrm{~F}=17,29$ & $\mathrm{~F}=20,73$ \\
& $\mathrm{P}<0,001$ & $\mathrm{P}<0,001$ & $\mathrm{P}<0,001$ \\
\hline
\end{tabular}

\pm S: Erreur standard; sur une même ligne et dans une même colonne, les moyennes suivies de la même lettre ne sont pas significativement différentes

Tableau 2 : Effet de la taille et du type d'explant sur le poids sec des cals chez le cotonnier.

\begin{tabular}{lccc}
\hline \multirow{2}{*}{$\begin{array}{l}\text { Taille des } \\
\text { explants }\end{array}$} & \multicolumn{3}{c}{ Pourcentage d'induction de cals } \\
\cline { 2 - 4 } & \multicolumn{3}{c}{ Type d'explant } \\
\cline { 2 - 4 } & Hypocotyle & Racine & Cotylédon \\
\hline $\mathbf{P}_{4}$ & $19,10 \pm 1,09^{\mathbf{a}}$ & $8,18 \pm 0,98^{\mathbf{c}}$ & $1,30 \pm 0,17^{\mathbf{e}}$ \\
P46 $_{\text {P6 }}$ & $11,72 \pm 1,10^{\mathbf{b}}$ & $7,26 \pm 0,52^{\mathbf{c}}$ & $2,83 \pm 0,55^{\text {ed }}$ \\
\hline Statistiques & $7,88 \pm 0,82^{\mathbf{c}}$ & $4,15 \pm 0,61^{\mathbf{d}}$ & $4,20 \pm 0,30^{\mathbf{d}}$ \\
& $\mathrm{F}=45,23$ & $\mathrm{~F}=11,78$ & $\mathrm{~F}=32,87$ \\
& $\mathrm{P}<0,001$ & $\mathrm{P}<0,001$ & $\mathrm{P}<0,001$ \\
\hline
\end{tabular}

$\pm \mathrm{S}$ : Erreur standard ; sur une même ligne et une même colonne, les moyennes suivies de la même lettre ne sont pas significativement différentes.

\section{DISCUSSION}

Les plantules obtenues après sept jours de germination in vitro présentent une hétérogénéité de taille. La germination des graines s'est effectuée dans les mêmes conditions, il est probable que la différence observée entre la taille des plantules soit liée à des facteurs endogènes aux graines. Heller et al. (1995) ont montré que l'état physiologique des graines et leur composition en hormone de croissance influencent la taille des plantules. Aussi, plusieurs auteurs ont noté que la croissance de l'hypocotyle et des racines est inhibée par une augmentation de la teneur des hormones endogènes, notamment les auxines et les cytokinines (Miller et Skoog, 1957; Vanderhoef et al., 1973 ; Fosket et al., 1981; Eliasson et al., 1989 ; Boyé, 2000). De même, Satchivi (1990) a rapporté que la masse des graines a une influence sur l'hétérogénéité de la taille des plantules de cotonnier.

Notre étude a montré que la callogenèse est meilleure avec l'hypocotyle qu'avec la racine suivie du cotylédon. Ces résultats sont en accord avec ceux de plusieurs auteurs qui ont montré que chez le cotonnier, l'hypocotyle est plus favorable à la callogenèse que la racine et le cotylédon (Peeters et Swennen, 1993; Koné, 1996 ; Zhang et al., 2001). La source de l'explant, et probablement sa structure anatomique semble jouer un rôle important dans l'induction des cals chez le cotonnier. Ce rôle de la source de l'explant dans l'initiation des cals a été aussi signalé chez de nombreuses plantes (Profumo et al., 1986 ; Fotso et al., 2002 ; Zouine et El Hadrami, 2004). Cette spécificité des explants pourrait s'expliquer par une réactivité ou une sensibilité différentielle des tissus au milieu 
d'initiation des cals (Kazumitsu, 1996; Zouzou et al., 1997). Aussi, l'analyse du poids sec des cals, résultat de la croissance des cals, montre que la callogenèse est inversement proportionnelle à la taille des plantules. Ainsi, le lot de plantules $\mathrm{P}_{4}$ est plus callogénique que les lots de plantules $\mathrm{P}_{46}$ puis $\mathrm{P}_{6}$. Cette réponse différentielle des lots de plantules à la callogenèse semble être due d'une part au diamètre de l'hypocotyle plus gros et d'autre part à la longueur de la racine principale plus courte chez $\mathrm{P}_{4}$ que ceux chez $\mathrm{P}_{46}$ et $\mathrm{P}_{6}$. Les travaux de Koné (1996) ont montré que la callogenèse, se déroulant au niveau de l'assise sous épidermique, est plus importante là où cette couche est la plus épaisse. La différence de diamètre des hypocotyles observée au niveau des trois lots de plantules serait liée à des facteurs endogènes, notamment les auxines, qui inhiberaient l'élongation de l'hypocotyle et favoriseraient sa croissance en épaisseur. Ainsi, ces auxines endogènes additionnées à celle contenue dans le milieu de culture auraient une action bénéfique sur l'induction des cals, ce qui expliquerait la bonne aptitude à la callogenèse observée avec les explants hypocotyles du lot de plantules $\mathrm{P}_{4}$ contrairement aux deux autres lots de plantules (Trolinder et Goodin, 1988; Kouakou, 1996 ; Zouzou et al., 1997). Aussi, les travaux de Boyé (2000) ont montré que les cytokinines endogènes inhibent la croissance racinaire des plantules, ce qui entraîne une accumulation de ces composés dans les racines, augmentant ainsi leur sensibilité à la prolifération cellulaire. Il existerait donc une corrélation négative entre la croissance de l'hypocotyle, de la racine et l'initiation des cals. Par contre, au niveau des cotylédons, nos résultats montrent l'existence d'une corrélation positive entre la taille des plantules et la callogenèse. Ainsi, les cotylédons du lot de plantules $\mathrm{P}_{6}$ produisent un poids sec de cals plus important que ceux de $\mathrm{P}_{46}$ puis de $\mathrm{P}_{4}$. Cette réactivité différente des cotylédons au milieu de callogenèse pourrait s'expliquer par une disponibilité plus importante des hormones dans les plantules à croissance élevée que dans celles à faible croissance
(Fosket et al., 1981 ; Zouzou et al., 1997 ; Boyé, 2000). Les récepteurs des phytohormones des cotylédons issus du lot de plantules $P_{6}$ semblent être plus sensibles par rapport à ceux des cotylédons issus des lots de plantules $\mathrm{P}_{4}$ et $\mathrm{P}_{46}$. Tous ces résultas révèlent la complexité d'action des hormones dont les effets varient d'un explant et/ou d'une plante à une autre.

\section{Conclusion}

La croissance en longueur et en largeur des hypocotyles et des racines (taille) des vitroplants est influencée par l'état hormonal de chaque graine de cotonnier. Plus la croissance des vitroplants est faible plus les potentialités callogéniques des explants hypocotyle et racine augmentent. En ce qui concerne les cotylédons, il est souhaitable d'utiliser ceux provenant des vitroplants de grande taille. La callogenèse est meilleure avec les explants issus des hypocotyles qu'avec ceux provenant respectivement des racines et des cotylédons.

\section{REFERENCES}

Anonyme. 2000. Cotton Magazine : Revue de la filière cotonnière ivoirienne. Actualité de la filière, $\mathrm{N}^{\circ} 11$, Décembre, $26 \mathrm{p}$.

Anonyme. 2006. Afrique agriculture: le programme de réforme foncière renforcé. Mensuel d'informations sur l'agriculture, l'élevage, la pêche et la forêt en Afrique, $\mathrm{N}^{\circ} 349$, juillet-août, $46 \mathrm{p}$.

Blanc G, Larder L, Martin A, Jacob JL, Carron MP. 2002. Differential carbohydrate metabolism conducts morphogenesis in embryogenic callus of Hevea brasiliensis (Müll. Arg). J. Exp. Bot., 53(373): 1453-1462.

Boyé MAD. 2000. Optimisation de la callogenèse par germination in vitro avec hormones chez le cotonnier (Gossypium hirsutum L. Var. ISA FMG 11). Mémoire de D.E.A, Physiologie végétale, Université d'Abidjan-Cocody (Côte d'Ivoire), p. 42

Démol J, Mergeai G, Hofs JL, Ndungo V. 1992. L'amélioration du cotonnier. In $L e$ 
Cotonnier au Zä̈re (vol 29). Publ. Agric. AGCD: Bruxelles, Belgique; 92-123.

Eliasson LG, Bertell C, Bolander E. 1989. Inhibitory action of auxin, on root elongation, not mediated by ethylen. Plant Physiol., 91: 310-314.

Finer JJ, Mc Mullen MD. 1990. Transformation of cotton (Gossypium hirsutum L.) via particle bombardment. Plant Cell Rep., 8: 586-589.

Fosket DE, Morejohn LC, Westernling KE. 1981. Control of growth by cytokinin. An examination of tubulin synthesis during cytokinin induced growth in cultured cell of Paul's scarlet rose. In Metabolisme and Molecular Activities of Cytokinins, Guera Peaud-Lenoel C (eds). Springer-Verlag: Berlin, Germany ; 193-211.

Gamborg OL, Miller RA, Ojima K. 1968. Nutrient requirements of suspension cultures of soybean root cells. Exp. Cell Res., 50: 151-158.

Gonzalez-Benito ME, Frota-Chagas JM, Peres C. 1997. Somatic embryogenesis of an early cotton cultivar. Pesqui. Agr. Bras., 32: 485-488.

Heller R, Esnault R, Lance C. 1995. Physiologie Végétale, Développement (Tome 2). Masson: Paris (France).

Kazutmitsu M. 1996. Callus induction and plantlet formation through culture of isolated microspores of eggplant (Solanum megalongena L.). Plant Cell Rep., 10: 319-325.

Koné M. 1996. Influence: Condition de culture, position explant hypocotyle et variété sur la callogenèse chez le cotonnier (Gossypium hirsutum L.). Mémoire de D.E.A, Physiologie végétale, Université d'Abidjan-Cocody (Côte d'Ivoire), p. 38.

Kouadio YJ, Koné M, Djè Y, D’Almeida MA, Zouzou M. 2004. L'étiolement est un facteur d'induction de l'embryogenèse somatique au cours de la callogenèse chez deux variétés récalcitrantes de cotonnier (Gossypium hirsutum L.) cultivées en Côte d'Ivoire. Biotechnol. Agron. Soc. Env., 8(3): 155-162.
Kouakou TH. 1996. Effet composition du milieu et régime hormonal sur la callogenèse chez le cotonnier (Gossypium hirsutum L. cv. ISA 205N). Mémoire de D.E.A, Physiologie végétale, Université d'Abidjan-Cocody (Côte d'Ivoire), p. 26.

Kouakou TH. 2009. Embryogenèse somatique chez le cotonnier [Gossypium hirsutum L. (malvaceae)] : variation des composés phénoliques au cours de la callogenèse et de la culture des suspensions cellulaires. Thèse de doctorat d'état, UFR des Sciences de la Nature, Laboratoire de Biologie et Amélioration des Productions Végétales, Université d'Abobo-AdjaméAbidjan (Côte d'Ivoire), p. 149.

Kouakou TH, Kouadio YJ, Koné M, Zouzou M, Anno AP. 2004. Evolution des composés phénoliques au cours de la callogenèse et de la culture de suspensions cellulaires chez Gossypium hirsutum L. Bioterre, 4(1): 5-13.

Kouakou TH, Waffo TP, Kouadio YJ, Valls J, Decendit A, Merillon JM. 2006. First evidence of trans-resveratrol production in cell suspension cultures of cotton. Plant Cell Tiss. Org. Cult., 26: 82-87.

Mergeai G. 1992. Utilisations du cotonnier sauvage Gossypium areysianum (DEFL.) HUTCH. Pour l'amélioration de l'espèce cultivée Gossypium hirsutum L. Thèse de doctorat, Phytotechnie des régions chaudes, Faculté des Sciences Agronomiques de Gembloux (Belgique), p. 180.

Miller CO, Skoog F, 1957. Chemical regulation of growth and organ formation in plant tissue cultivated in vitro. Exp. Biol., 11: 118-130.

Munro JM. 1987. Cotton. Trop. Agri., 2: 170172.

Murashige T, Skoog F. 1962. A revised medium for rapid growth and bioassays with tobacco tissue cultures. Physiol. Plant., 15: 473-497.

Olesen A, Storgaard M, Folling M, Madison S, Andersend S. 1995. Protoplast, Callus and Suspension Culture of Perennial 
ryegrass. Kluwer Acad. Publ. : Netherland.

Satchivi N. 1990. Effet sur la germination des graines de cotonnier de dérivés indolylcarboxyliques. Mémoire de D.E.A, Physiologie végétale, Université d'Abidjan-Cocody (Côte d'Ivoire), p. 63

Peeters MC, Swennen R. 1993. Cotton biotechnology, State of Art. Proceeding of $1^{\text {st }}$ African Crop Science Conference, African Crop Science Society Eds. Kampala (Uganda), 1-16.

Profumo P, Gastaldo P, Dameri R, Caffaro L. 1986. Histological study of calli and embryoïdes from explants of Aesculus hippocastanum L. Plant Physiol., 126: 97-103.

Sément G. 1986. Le Cotonnier en Afrique Tropicale. Le Technicien d'Agriculture Tropicale. Maisonneuve et Larose : Paris, France.

Trolinder NL, Goodin JR. 1988. Somatic embryogenesis and plant regeneration in cotton (Gossypium hirsutum L.). Effect of source of explant and hormonal regime. Plant Cell Tiss. Org. Cult. 12: 31-42.

Vaissayre M, Lançon J. 1991. Protection des cotonniers sans glandes: Bilan de dix années d'expérimentation en Côte d'Ivoire (1981-1990). Commission régionale de défense des cultures Afrique de l'Ouest, Doc. IRCT-CIRAD, France, $160 \mathrm{p}$.
Vanderhoef LN, Stahl C, Siegel N, Zeigler R. 1973. The inhibition by cytokinin of auxin-promoted elongation in excised soybean hypocotyls. Physiol. Plant, 29: 22-27.

Zhang BH, Feng R, Liu F, Wang Q. 2001. High frequency somatic embryogenesis and plant regeneration of an elite Chinese cotton variety. Bot. Bull. Acad. Sin., 42: 9-16.

Zouine J, El Hadrami I. 2004. Somatic Embryogenesis in Phoenix dactylifera L.: Effect of Exogenous Supply of Sucrose on Proteins, Sugar, Phenolics and Peroxydases Activities during the Embryogenic Cell Suspension Culture. Biotechnology, 3(2): 114- 118.

Zouzou M, Kouakou T, Koné M, Peeters M, Swennen R. 1997. Callogenèse chez le cotonnier cultivé en Côte d'Ivoire : effets position explant hypocotyle, variétés, source de carbone et régime hormonal. In Proceeding of $3^{\text {rd }}$ African Crop Science Conference, African Crop Science Society eds. Pretoria (Afrique du Sud), 1489-1494.

Zouzou M, Kouadio Y, Koné M, Kouakou T, Dénézon D. 2000. Callogenèse chez Gossypium hirsutum L. : effets cultivar, conditions de culture et type de matériel. Bioterre, 1(1): 48-56. 